\title{
Validation of the Brazilian Portuguese version of the Copenhagen Burnout Inventory (CBI) in healthcare professionals
}

Carolina M. Moser; Bárbara Tietbohl-Santos; Daniel Luccas Arenas; Aurora Xavier; Felipe Ornell; Rogerio Boff Borges; Simone Hauck.

The authors have withdrawn this manuscript because they are working with the author of the original Copenhagen Burnout Inventory (CBI) on additional analyses that they want to include. Their study is part of a larger one (a cohort study), which allows the analysis of the test-retest reliability of the instrument that they also want to include. Therefore, the authors do not wish this work to be cited as reference for the project. If you have any questions, please contact the corresponding author. 